\title{
Correction to: $\mathrm{CT}$ and MR imaging prior to transcatheter aortic valve implantation: standardisation of scanning protocols, measurements and reporting-a consensus document by the European Society of Cardiovascular Radiology (ESCR)
}

\author{
Marco Francone $^{1}$ (D) $\cdot$ Ricardo P. J. Budde ${ }^{2}$. Jens Bremerich ${ }^{3} \cdot$ Jean Nicolas Dacher ${ }^{4} \cdot$ Christian Loewe $^{5} \cdot$ Florian Wolf $^{5}$. \\ Luigi Natale $^{6} \cdot$ Gianluca Pontone $^{7} \cdot$ Alban Redheuil $^{8,9,10} \cdot$ Rozemarijn Vliegenthart $^{11} \cdot$ Kostantin Nikolaou $^{12}$. \\ Matthias Gutberlet ${ }^{13} \cdot$ Rodrigo Salgado $^{14,15}$
}

Published online: 2 March 2020

(C) The Author(s) 2020

\section{Correction to: European Radiology}

https://doi.org/10.1007/s00330-019-06357-8

The original version of this article, published on 05 September 2019 , unfortunately contained a mistake. The image of "Jena Valve" in Table 3 was incorrect. The corrected table is given below.

The online version of the original article can be found at https://doi.org/ 10.1007/s00330-019-06357-8

Marco Francone

marco.francone@uniroma1.it

1 Department of Radiological, Oncological and Pathological Sciences, Sapienza University, Policlinico Umberto I, V.le Regina Elena 324, 00161 Rome, Italy

2 Department of Radiology \& Nuclear Medicine, Erasmus MC, Rotterdam, The Netherlands

3 Department of Radiology, University of Basel Hospital, Basel, Switzerland

4 Department of Radiology, Normandie University, UNIROUEN, INSERM U1096 - Rouen University Hospital, F 76000 Rouen, France

5 Division of Cardiovascular and Interventional Radiology, Department of Biomedical Imaging and Image-Guided Therapy, Medical University of Vienna, Vienna, Austria

6 Department of Radiological Sciences - Institute of Radiology, Catholic University of Rome, "A. Gemelli” University Hospital, Rome, Italy
7 Centro Cardiologico Monzino, IRCCS, Milan, Italy

8 Institute of Cardiometabolism and Nutrition (ICAN), Paris, France

9 Department of Cardiovascular and Thoracic, Imaging and Interventional Radiology, Institute of Cardiology, APHP, Pitié-Salpêtrière University Hospital, Paris, France

10 Laboratoire d'Imagerie Biomédicale, Sorbonne Universités, UPMC Univ Paris 06, INSERM 1146, CNRS, 7371 Paris, France

11 Department of Radiology, University of Groningen, University Medical Center Groningen, Groningen, Netherlands

12 Department of Diagnostic and Interventional Radiology, University of Tuebingen, Tübingen, Germany

13 Diagnostic and Interventional Radiology, University of Leipzig-Heart Center, Leipzig, Germany

14 Department of Radiology, Antwerp University Hospital, Antwerp, Belgium

15 Department of Radiology, Holy Heart Hospital, Lier, Belgium 
Table 3 Overview of new generation TAVI devices

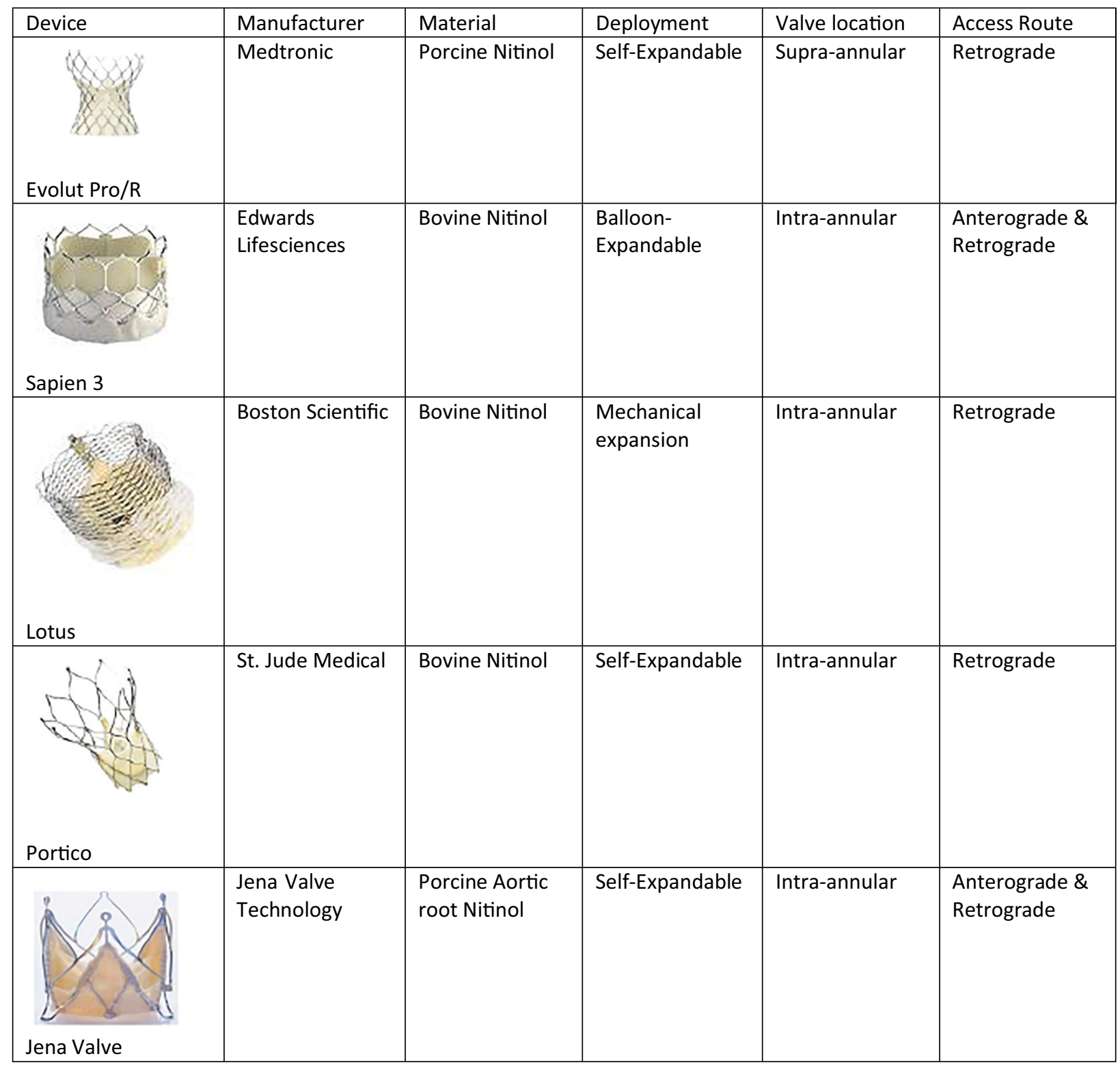

Open Access This article is distributed under the terms of the Creative Commons At tribution 4.0 International License (http:/ / creativecommons.org/licenses/by/4.0/), which permits unrestricted use, distribution, and reproduction in any medium, provided you give appropriate credit to the original author(s) and the source, provide a link to the Creative Commons license, and indicate if changes were made.
Publisher's note Springer Nature remains neutral with regard to jurisdictional claims in published maps and institutional affiliations. 